\title{
A RELAÇÃO ENTRE O GÊNERO E A EXPRESSÃO DO TEMPERAMENTO
}

Luiz Antônio Ferreira Cavalcante Dutra, Universidade de Rio Verde, luizjacy@ hotmail.com.

Claudio Herbert Nina-e-Silva, Laboratório de Psicologia Anomalística e Neurociências, Universidade de Rio Verde.

\section{Recebido em: 28/03/2014 - Aprovado em: 30/06/2014 - Disponibilizado em: 30/07/2014}

Resumo: O presente trabalho teve como objetivo verificar se há diferença entre mulheres e homens quanto à expressão do temperamento. Participaram do estudo 48 estudantes universitários ( 25 mulheres e 23 homens). O temperamento dos participantes foi avaliado por meio da escala BIS/BAS baseada na Teoria da Sensibilidade ao Reforço de Gray. Os resultados indicaram a existência de diferença de temperamento entre os gêneros. Os homens apresentaram mais freqüência de ocorrência do temperamento "ansioso", enquanto que não houve predomínio de nenhum tipo de temperamento entre as mulheres estudadas.

Palavras-chave: temperamento, personalidade, gênero, BIS/BAS, psicobiologia.

Abstract: This study aimed to verify whether there are differences between women and men for the expression of temperament. Study participants were 48 college students ( 25 women and 23 men). The temperament of the participants was assessed by BIS / BAS scale based on the Reinforcement Sensitivity Theory of Gray. The results indicated the existence of differences in temperament between genders. Men showed greater frequency of occurrence of the "Anxiety" temperament, whereas there was no predominance of any temperament among the women studied.

Keywords: temperament, personality, gender, BIS/BAS, psychobiology.

\section{Introdução}

O temperamento pode ser definido como a forma característica, de base biológica, de uma pessoa reagir ao ambiente físico e/ou social que a rodeia (ROTHBART; BATES, 1998; ROTHBART; AHADI; EVANS, 2000).

Portanto, o conceito de temperamento se refere às diferenças individuais constitucionais (psicofisiológicas) na forma de expressar e de autorregular as emoções (ROTHBART; BATES, 1998). Desse modo, o temperamento pode ser considerado a base emocional da personalidade (CARVER; WHITE, 1994; ROTHBART; AHADI; EVANS, 2000; CORR; 2002; KAIL, 2004).

Nesse sentido, de acordo com Bee (2003, p.658), "o temperamento é a matriz da qual se desenvolve posteriormente a personalidade da criança e do adulto".
Os estudos longitudinais de personalidade com gêmeos têm evidenciado a base genética do temperamento (KAY; TASMAN, 2001). A genética influenciaria no temperamento por meio da configuração estrutural do sistema nervoso e da predisposição a modos específicos de funcionamento neurofisiológico (KAGAN; REZNICK; CLARKE; SNIDMAN; GARCIA-COLL, 1984; KAGAN; REZNICK; SNIDMAN, 1987).

A Teoria da Sensibilidade ao Reforço do psicólogo inglês Jeffrey Gray propõe uma análise do temperamento humano a partir de duas dimensões básicas relacionadas ao funcionamento do sistema nervoso central: 1) Sistema de Ativação Comportamental, BAS na sigla em inglês; e 2) Sistema de Inibição Comportamental, BIS na sigla em inglês (CARVER; WHITE, 1994; CORR, 2002;

TULL; GRATZ; LATZMAN; KIMBREL; 
LEJUEZ, 2010). Enquanto o BAS está relacionado ao temperamento impulsivo e à sensibilidade ao reforço, o BIS diz respeito ao temperamento ansioso e à sensibilidade à punição.

$\mathrm{O}$ estudo do temperamento tem sido considerado fundamental para o entendimento das diferenças psicológicas entre os gêneros, pois, de modo geral, a literatura não apresenta consenso quanto à existência ou não de diferenças significativas de temperamento entre bebês meninos e meninas, embora, também, há controvérsia sobre as evidências de diferenças de temperamento entre homens e mulheres adultos (BEE, 2003; KAIL, 2004; PAPALIA; FELDMAN; OLDS, 2008; ELSEQUEST; HYDE; GOLDSMITH; VAN HULLE, 2006; TULL et al., 2010).

A adequada compreensão das semelhanças e das diferenças entre os gêneros seria importante para o planejamento de ações educativas e de intervenção psicológica. Além disso, o conhecimento de eventuais diferenças de temperamento entre os gêneros contribuiria para diminuir o preconceito de gênero e minimizar o sexismo.

Desse modo, o objetivo do presente trabalho foi descrever a relação entre o gênero e a expressão do temperamento.

\section{Materiais e Métodos}

\subsection{Participantes}

Participaram deste estudo 48 estudantes universitários do curso de Psicologia de uma faculdade da cidade de Rio Verde-GO.

\subsection{Instrumentos}

A avaliação do temperamento dos participantes foi feita por meio da Escala BIS/BAS (CARVER; WHITE, 1994), traduzida e adaptada para a realidade brasileira por Portilho-Souza e Nina-e-Silva (2013). A Escala BIS/BAS é composta por 24 itens, sendo que o participante classificou cada um desses itens de acordo com uma escala tipo Likert que apresenta quatro pontos: $1=$ muito verdadeiro para mim; $2=$ mais verdadeiro do que falso para $\mathrm{mim} ; 3=$ mais falso do que verdadeiro para mim; 4=totalmente falso para mim.

\subsection{Procedimento}

Após a aprovação do Comitê de Ética em Pesquisa da Universidade de Rio Verde (parecer $\mathrm{n}^{\mathrm{o}}: 138 / 2012$ e registro $\left.\mathrm{n}^{\mathrm{o}}: 021 / 2012\right)$, iniciou-se o recrutamento dos participantes. Explicou-se a eles o objetivo da pesquisa e como seria a participação deles.

Depois da leitura e assinatura do Termo de Compromisso Livre e Esclarecido, os participantes responderam, individualmente, em sala de aula destinada para esse fim, à Escala BIS/BAS. 


\subsection{Análise dos dados}

A verificação da relação entre os escores de temperamento da escala BIS/BAS e a variável de gênero foi feita através do cálculo do teste $t$ de diferença entre médias presumindo variâncias diferentes por meio do aplicativo Microsoft Excel 2010.

\section{Resultados e Discussão}

O Quadro 1 mostra a distribuição percentual dos participantes dos gêneros feminino e masculino nos tipos de temperamento a partir do cálculo dos escores obtidos na Escala BIS/BAS. De modo geral, observou-se que praticamente não houve diferença na distribuição das participantes do gênero feminino entre os tipos de temperamento Impulsividade e Ansiedade.

Quadro 1: Classificação dos participantes em tipos de temperamento por gênero (em \%).

\begin{tabular}{|c|c|c|}
\hline \multirow{2}{*}{ GÊNERO } & \multicolumn{2}{|c|}{ TEMPERAMENTO } \\
\cline { 2 - 3 } & ANSIEDADE & IMPULSIVIDADE \\
\hline FEMININO & 52 & 48 \\
\hline MASCULINO & 86,36 & 13,63 \\
\hline
\end{tabular}

Fonte: Os autores.

Esses resultados estão em desacordo com literatura. segundo a qual as mulheres tenderiam a apresentar predomínio do tipo de temperamento "Ansiedade" (CARVER; WHITE, 1994; WRIGHT; HARDIE; WILSON, 2009).

No caso dos participantes do gênero masculino, verificou-se que houve predomínio do tipo de temperamento “Ansiedade". Esse resultado está em desacordo com a literatura (CARVER;
WHITE, 1994; ELSE-QUEST et al., 2006; TULL et al., 2010) visto que, nesses estudos, a maioria dos homens demonstrou temperamento do tipo "Impulsividade".

Essa discrepância entre os presentes resultados para os participantes do gênero masculino e os resultados comumente descritos na literatura poderia ser explicada por um viés de amostra, uma vez que todos os participantes foram selecionados entre os alunos do curso de Psicologia. Castro e Yamamoto (1998) realizaram um estudo que evidenciou uma distribuição desigual de homens e mulheres em curso de Psicologia no Brasil, com a grande maioria dos acadêmicos pertencendo ao gênero feminino. Esses autores argumentaram que o predomínio de mulheres nos cursos de Psicologia no Brasil poderia ser explicado pela representação social da Psicologia no nosso país como tendo um "status de profissão feminina" (CASTRO; YAMAMOTO, 1998, p.154).

Dessa maneira, uma hipótese a ser verificada posteriormente seria a de que os homens que buscam fazer o curso de Psicologia poderiam apresentar um temperamento mais parecido com o das mulheres, isto é, o temperamento ansioso. Trata-se, portanto, de uma questão empírica que necessita da realização de novos estudos para investigá-la.

Observou-se diferença estatisticamente significativa entre os escores da escala BIS BAS obtidos por mulheres e homens ( $t=$ 3,118064, $\quad p=0,001707)$. Embora essa 
diferença de temperamento entre os gêneros tivesse sido prevista pela literatura (CARVER; WHITE, 1994; ELSE-QUEST et al., 2006; WRIGHT; HARDIE; WILSON, 2009; TULL et al., 2010), não havia previsão relativa ao fato de os homens terem apresentado mais predomínio do temperamento "Ansiedade" do que as mulheres

Quanto à questão da existência de diferença de temperamento entre homens e mulheres, existe uma controvérsia entre a literatura relacionada à psicologia do desenvolvimento e a literatura relacionada à Teoria da Sensibilidade ao Reforço. A literatura relacionada à psicologia do desenvolvimento (PAPALIA; FELDMAN; OLDS, 2008; KAIL，2004; BEE，2003) afirma que não haveria consenso sobre a existência de diferenças significativas de temperamento entre os gêneros. De acordo com o trabalho de revisão sistemática da literatura sobre as diferenças psicológicas entre os gêneros realizado por Poeschl, Múrias e Ribeiro (2003), não haveria evidências empíricas de que existam diferenças psicológicas significativas entre homens e mulheres.

Por outro lado, a literatura vinculada à Teoria da Sensibilidade ao Reforço tem demonstrado a existência de diferenças de temperamento entre os gêneros (CARVER; WHITE, 1994; ELSE-QUEST et al., 2006; WRIGHT; HARDIE; WILSON, 2009; TULL et al., 2010). Os presentes resultados que evidenciaram diferença significativa de temperamento entre os gêneros, portanto, estão em desacordo com a literatura vinculada à psicologia do desenvolvimento, mas corroboram os estudos anteriores que também utilizaram a escala BIS/BAS como instrumento de coleta de dados.

\section{Conclusão}

O presente estudo teve como objetivo geral verificar a existência de relação entre o gênero e a expressão do temperamento. Os resultados evidenciaram a existência de diferença estatisticamente significativa de temperamento entre os gêneros. Os homens apresentaram mais freqüência de ocorrência do temperamento ansioso, enquanto que não houve predomínio de nenhum tipo de temperamento entre as mulheres estudadas.

Sugere-se a realização de novos estudos que ampliem a amostra e evitem o viés dos participantes oriundos do curso de Psicologia.

\section{Referências Bibliográficas}

BEE, H. A criança em desenvolvimento. Porto Alegre: Armed, 2003.

CARVER, C.S.; WHITE, T.L. Behavioral inhibition, behavioral activation, and affective responses to impending reward and punishment: The BIS/BAS scales. Journal of Personality and Social Psychology, 67, p.319-333, 1994.

CASTRO, A.E.; YAMAMOTO, O. H. A psicologia como profissão feminina: apontamentos para estudo. Artigo. Doc. Rio 
Grande do Norte, 1998. Disponível em $<$ http.// www.scielo.br/pdf.epsic/vn3n1/pdf./ $>$ users acesso em 16-set-2012.

CORR, P.J. J. A. Gray's reinforcement sensitivity theory: tests of the joint subsystems hypothesis of anxiety and impulsivity. Personality and Individual Differences, 33, p.511-532, 2002.

ELSE-QUEST, N.M; HYDE, J.S.H.; GOLDSMITH, H.; VAN HULLE, C.A. Gender Differences in Temperament: A MetaAnalysis Psychological Bulletin, 132(1), p. 33-72, 2006.

KAGAN, J.;REZNICK, J.S.; CLARKE，C.; SNIDMAN, N.; GARCIA-COLL, C. Behavioral inhibition to the unfamiliar. Children Development, 55(22), p. 12-25, 1984.

KAGAN, J.;REZNICK, J.S; SNIDMAN, N. The physiology and psychology of behavioral inhibition in children. Children Development, 58(14), p. 59-73, 1987.

KAIL, R.V. A criança. São Paulo: Prentice Hall, 2004.

KAY, J; TASMAN, A. Psiquiatria: ciência comportamental e fundamentos clínicos. São Paulo: Manole, 2002.

PAPALIA, D. E.; FELDMAN, D.R.; OLDS, J.W. Desenvolvimento Humano. Porto Alegre: AMGH, 2008.

PORTILHO-SOUZA, E.; NINA-E-SILVA, C. H. Tradução e adaptação da escala BIS/BAS para aplicação em adultos brasileiros. Revista da Universidade Vale do Rio Verde, 11(2), p.470-476, 2013.

ROTHBART; M.K.; BATES, J.E. Temperament. Em: W. Damon; N. Einsenberg (Eds). Handbook of child psychology vol 3: social, emotional and personality development. , p.105-176. Nova Iorque: Wiley, 1998.
ROTHBART; M.K.; AHADI, S.A.; EVANS, D.E. Temperament and personality: origins and outcomes. Journal of Personality and Social Psychology, 78(1), p.122-135, 2000.

TULL, M.T.; GRATZ, K.L.; LATZMAN, R.D.; KIMBREL, N.A.; LEJUEZ, C.J. Reinforcement Sensitivity Theory and emotion regulation difficulties: A multimodal investigation Personality and Individual Differences, 49(1), p.989-994, 2010.

WRIGHT, L.; HARDIE, S.M; WILSON, K. Handedness and behavioural inhibition: Lefthanded females show most inhibition as measured by BIS/BAS self-report. Personality and Individual Differences, 46(1), p.20-24, 2009. 